\title{
Modern Possibilities of Metabolic Therapy in Improving the Quality of Life in Patients with Postinfarction Cardiosclerosis
}

\author{
Vasilieva Irina, Rezvan Vladimir, Dvoretskiy Leonid \\ I. M. Sechenov First Moscow State Medical University, Moscow, Russia \\ Email address: \\ emmans@rambler.ru (V. Irina), vladimir.rezvan@mail.ru (R. Vladimir), dvoretski@mail.ru (D. Leonid) \\ ${ }^{*}$ Corresponding author
}

\section{To cite this article:}

Vasilieva Irina, Rezvan Vladimir, Dvoretskiy Leonid. Modern Possibilities of Metabolic Therapy in Improving the Quality of Life in Patients with Postinfarction Cardiosclerosis. American Journal of Internal Medicine. Vol. 7, No. 4, 2019, pp. 86-92.

doi: 10.11648/j.ajim.20190704.12

Received: June 24, 2019; Accepted: July 16, 2019; Published: July 26, 2019

\begin{abstract}
II and III functional classes were examined. The study included patients who refused intervention revascularization. The main group included 48 patients who were added taurine (Dibikor $750 \mathrm{mg} /$ day) to enhance the effect of basic therapy, and the comparison group included 47 patients who received standard therapy and placebo. The duration of treatment was 3 months. The study demonstrated positive effects of taurine that was confirmed by significant improvements in the following indicators: subjective status, indicators of the Seattle questionnaire, echocardiographic parameters, cardiac arrhythmias. There was a decrease in the severity of fatigue by 2 times, a decrease in the intensity of complaints of palpitations by $72.3 \%$, a decrease in the severity of dyspnea by $30.0 \%$ and a severity of pain in the heart area by $50 \%$. Indicators of most scales of the Seattle questionnaire in patients of the main group at the end of the study significantly exceeded $(\mathrm{p}<0.05)$ the corresponding values in patients of the comparison group. In patients whose treatment was used taurine, a significant improvement in inotropic function was noted (the ejection fraction is significantly higher than in the comparison group, respectively $56.0 \pm 1.8$ and $53.5 \pm 1.1 \%, \mathrm{p}<0,05$ ), the bathmotropic and chronotropic myocardial functions improved, which was shown by a $45 \%$ decrease in the number of ventricular extrasystoles, a $57 \%$ decrease in the frequency of supraventricular heart rhythm disturbances, a decrease in the incidence rate of ST segment depression (in the main group the number of episodes was significantly lower than $p<0,05$ than in the comparison group). The use of taurine in the complex treatment of patients with exertional angina, after suffering a myocardial infarction, is safe and not accompanied by side effects. Taurine is advisable to include in the complex treatment of patients with exertional angina pectoris, who have had a myocardial infarction in order to improve the myocardial inotropic function, enhance the antiarrhythmic and lipid-lowering effects of the basic therapy. The recommended dose of Taurine is $750 \mathrm{mg}$ per day as a supplement to standard therapy with a duration of therapy of at least 3 months.
\end{abstract}

Keywords: Angina, Postinfarction Cardiosclerosis, Taurine, Quality of Life, Exercise Tolerance, Cardiac Arrhythmias

\section{Introduction}

The problem of postinfarction cardiosclerosis is reflected in many recent scientific papers. The basis of these studies is the study of the characteristics of hemodynamics and the state of structural geometric cardiac remodeling, neurovegetative regulation, electrophysiological signs of electrical instability, cardiac arrhythmias, dispersion of the QT interval, the presence of late ventricular potentials [1-3]. A number of works are devoted to the search for effective and safe methods of treatment and prevention of the above described deviations [3, 4], while the use of taurine [4-6] is considered as a promising approach in this aspect.

To date, a number of studies in a high degree of accuracy have shown an inverse correlation between the consumption of taurine in the composition of food and the risk level of cardiovascular accidents, as well as mortality from cardiovascular diseases $[4,7]$. The participation of this 
substance in the regulation of intracellular calcium metabolism is shown, because taurine is a calcium antagonist, affects the sensitivity of contractile proteins to calcium, inhibits apoptosis, has a protective effect on myocardial cells. There is evidence of its effect on the level of adenosine triphosphate [5,8]. Thus, the results of a numerous of studies have demonstrated the perspective for the use of taurine in the prevention and treatment of cardiovascular and other diseases. However, reports on the possibility of using this drug in patients with postinfarction cardiosclerosis are not systematized, and there are no common views on the feasibility of including taurine in the complex of therapeutic measures in this cohort of patients. In this regard, current research on assessing the clinical efficacy and safety of this drug, its impact on the clinical, instrumental and laboratory characteristics of coronary heart disease, the quality of life of these patients, including in the long-term period after the end of taking taurine.

\section{Material and Methods}

The study included 95 patients with postinfarction cardiosclerosis and angina of the II and III functional classes. A placebo-controlled randomized trial was conducted. The diagnosis of the underlying disease is made on the basis of complaints, anamnesis, clinical symptoms, instrumental data: electrocardiograms (ECG), echocardiography (Echo-KG), exercise tests, Holter monitoring.

Exclusion criteria from the study were: cardiac arrhythmias and conduction (atrial fibrillation, sustained ventricular tachycardia), atrioventricular block II-III degrees, complete blockade branches of His bundle, myocardial revascularization, chronic heart failure II B-III stage, pregnancy, breastfeeding, diabetes.

After signing the informed consent, the patients were randomly divided into two groups:

a) the main group consisted of 48 patients (29 men and 19 women, average age $65.8 \pm 7.2$ years), to whom taurine was added to the standard therapy (Dibikor, $750 \mathrm{mg} /$ day);

b) comparison group - 47 patients (30 men and 17 women, mean age $63.6 \pm 6.9$ years) who received standard therapy and placebo. The duration of treatment was 3 months.

Comparison of the patients of the main group and the comparison group before the start of treatment showed that, according to clinical manifestations, results of instrumental and laboratory studies, there are no significant intergroup differences. Patients were included in the study in a stable state, they received standard therapy recommended by the European Society of Cardiology for the treatment of coronary artery disease and chronic heart failure: angiotensin-converting enzyme inhibitors, b-blockers, aldosterone antagonists, clopidogrel, acetylsalicylic acid, atorvastatin. According to indications, thiazide or loop diuretics were prescribed. Therapy was not changed through the observation period.
Before the start of the study, after 3 and 6 months from the beginning of the study, a general assessment of the clinical status of patients in the main group and the comparison group was performed. As part of the study, patients complaints about fatigue, heartbeat, heart failure, shortness of breath, and heart pain were taken into account. For each complaint the patient ranked on a five-point scale from 0 (not worried) to 5 (very worried).

ECG registration was performed in 12 leads (speed $25 \mathrm{~mm}$ / s) for all patients on the "Cardovit AT-2" apparatus (Schiller, Switzerland). Holter ECG monitoring was performed daily for all patients using the automatic monitoring system "MT-100" (Schiller, Switzerland, twochannel cable) under normal motor conditions for 24 hours. The presence of signs of ischemia, the dynamics of patients with heart rhythm disorders were assessed by the number of ventricular and supraventricular extrasystoles for 24 hours.

Assessment of exercise tolerance was performed using the "MTM-1500" apparatus (Schiller, Switzerland). Patients underwent treadmill test using a modified Bruce protocol. According to the test results, the number was estimated metabolic equivalents (MET) for each patient.

Echocardiography was performed using the "VIVID 4" device (GE Healthcare, United Kingdom). The sizes of the left atrium, right atrium, left ventricular ejection fraction, stroke volume, shortening fraction, thickness of the posterior wall of the left ventricle, interventricular septum, endsystolic size, enddiastolic size, endsystolic volume, enddiastolic volume.

When assessing the quality of life of patients the Seattle Questionnaire was used developed by Spertus J and et al. [9]. This questionnaire includes 19 questions combined in subsections: 9 questions on exercise tolerance, 1 question on the stability of angina, 2 questions on the frequency of angina, 4 questions on satisfaction with treatment and 3 questions on the perception of the disease. Depending on the patient's response, a rating was given from 1 to 5 points for each question, and then the total score was calculated.

Statistical data processing was performed using the computer software STATISTICA 10 for Windows (Stat Soft, USA). For all quantitative parameters studied, mean values and standard errors of the mean were calculated. The normal distribution of the indicators in the groups was checked using the Kolmogorov-Smirnov criterion. In assessing the statistical significance of the differences between the studied groups, the parametric Student's t-test (for samples with a proven normal distribution of values) and the non-parametric Mann-Whitney test were used in all other cases. To assess the statistical significance of differences between the indices of one group at different stages of observation, the Student's t-test was used for related samples (for samples with a proven normal distribution of values) and the Wilcoxon test (for samples with an abnormal distribution). To determine differences in the frequency of manifestations of symptoms in groups of examined patients, the indicator $\%$ was calculated. Differences were considered significant at $p$ $<0.05$. 


\section{Results}

After treatment the patients of the main group revealed a statistically significant decrease in the severity of fatigue almost twice - after 3 months from the start of treatment, from $1.52 \pm 0.14$ to $0.83 \pm 0.10$ points, $\mathrm{p}<0.0005)$, which persisted after 6 months from the beginning of the study -
$0.71 \pm 0.08$ points (Figure 1 ). In the comparison group, the decrease in the expression of complaints of fatigue was significant less pronounced, the values of the indicator were in these terms, respectively $1.30 \pm 0.10$ and $1.19 \pm 0.10$ points, which was significantly higher in both cases ( $p$ $<0.05)$ than in patients in the treatment which used taurine.

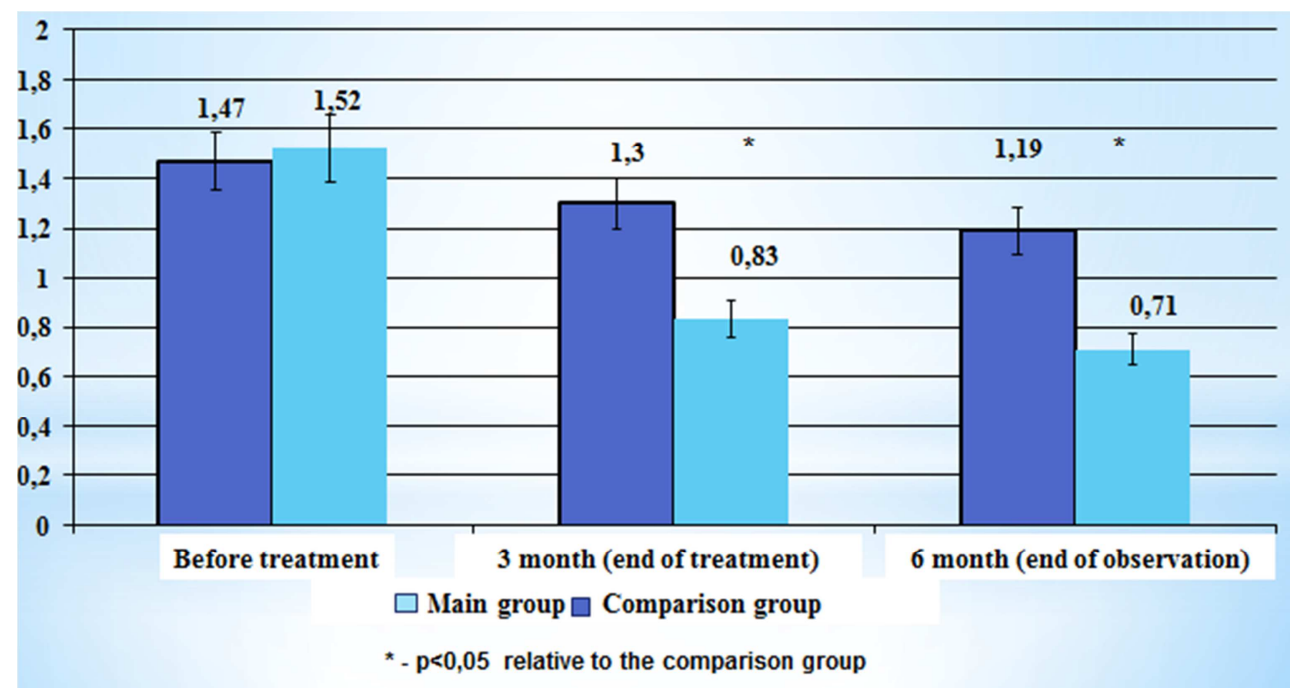

Figure 1. The dynamic of complaints on fatigue in the main and the comparison group.

The intensity of palpitations in the group of patients taking taurine decreased in the first 3 months by $73 \%$ (from $0.48 \pm$ 0.07 to $0.13 \pm 0.05$ point, $\mathrm{p}<0.001$ ). In the comparison group the statistically significant dynamics of palpitations during the first 3 months. The value of this indicator was actually at the same level, reaching $0.51 \pm 0.10$ before the start of treatment and $0.53 \pm 0.09$ after 3 months, which was significantly higher $(\mathrm{p}<0.05)$ than in patients taking taurine. After 6 months from the start of treatment in the main group, the severity of palpitations was $0.19 \pm 0.06$ points, which was significantly lower than before the start of treatment ( $p$ $<0.02$ ). In patients taking placebo, after 6 months after the start of the study, the intensity of palpitations complaints remained at the same level $(0.55 \pm 0.09)$, which was significantly $(\mathrm{p}<0.05)$ higher than in the main group.

Patients of the main group after 3 months after initiation of therapy significantly less frequently $(p<0.05)$ than patients in the comparison group presented other complaints, such as interruptions in heart function, shortness of breath and pain in heart. So, after the treatment, the average severity of complaints of heart pain in patients of the main group significantly decreased by more than $50 \%$ (from $0.65 \pm 0.10$ to $0.38 \pm 0.07$ points, $p<0.001$ ), the value of this parameter remained reduced and at the end of the observation - after 6 months, reaching $0.42 \pm 0.07$ points. In patients from the comparison group, the value of this indicator after 3 months increased from $0.70 \pm 0.60$ to $0.77 \pm 0.10$ points, further decreased to $0.66 \pm 0.08$ points, while not significantly different from the initial level and was significantly higher ( $\mathrm{p}$ $<0.01$ ) than in the main group.

Evaluation quality of life of patients using the Seattle questionnaire showed that after 3 months after the start of therapy, the indices of patients taking taurine increased significantly on most scales (Table 1). Thus, a significant ( $p$ $<0.05$ ) increase in the indicator "limitation of physical activity" (PL) was found to improve exercise tolerance in patients of both groups, while in patients of the main group the value of this indicator was significantly $(\mathrm{p}<0.05)$ higher than in the comparison group.

Table 1. Dynamics indicators quality of life (Seattle Questionnaire), $M \pm m$ $(n=95)$.

\begin{tabular}{lll}
\hline Term & Comparisongroup $(\mathbf{n}=\mathbf{4 7})$ & Main group $(\mathbf{n}=\mathbf{4 8})$ \\
\hline \multicolumn{2}{l}{ Scale PL (Limit physical activity) } & \\
Before treatment & $34.4 \pm 1.0$ & $35.1 \pm 1.8$ \\
After 3 months & $39.3 \pm 1.4$ & $48.3 \pm 2.0 * \#$ \\
After 6 months & $37.2 \pm 1.3$ & $49.0 \pm 1.8 * \#$ \\
Scale AS (Stability of angina) & \\
Before treatment & $38.8 \pm 4.2$ & $40.1 \pm 3.7$ \\
After 3 months & $42.6 \pm 3.4$ & $51.3 \pm 3.1 * \#$ \\
After 6 months & $40.4 \pm 3.2$ & $48.4 \pm 3.0 * \#$ \\
Scale AF (Frequency of Angina) & \\
Before treatment & $35.3 \pm 2.2$ & $36.3 \pm 2.3$ \\
After 3 months & $39.8 \pm 2.6$ & $48 \pm 3.4 * \#$ \\
After 6 months & $38.9 \pm 2.5$ & $45.4 \pm 2.7 * \#$ \\
TS Scale (Treatment Satisfaction) & \\
Before treatment & $33.1 \pm 1.4$ & $34.8 \pm 2.3$ \\
After 3 months & $38.6 \pm 1.9$ & $47.6 \pm 2.5 * \#$ \\
After 6 months & $35.4 \pm 1.3$ & $48.5 \pm 2.3 * \#$ \\
DP Scale (Disease Perception) & \\
Before treatment & $36.2 \pm 2.3$ & $37.0 \pm 2.1$ \\
After 3 months & $39.7 \pm 2.4$ & $47.9 \pm 2.1 * \#$ \\
After 6 months & $35.4 \pm 2.0$ & $42.5 \pm 1.9 * \#$ \\
\hline
\end{tabular}

Table 1

Note: * - differences are significant (at $\mathrm{p}<0.05$ ) relative to the indicator of the comparison group (cr. Mann-Whitney), \# - differences are significant (at $\mathrm{p}<0.05$ ) relative to the level before treatment (cr. Wilcoxon). 
After the end of treatment an increase in the levels of indicators of this questionnaire was also revealed: scales "stability of angina pectoris", "frequency of stenocardia", "satisfaction with treatment". The levels of these indicators in the main group increased relative to baseline data, respectively, by $30-58 \%$.

In the comparison group after 6 months after the start of treatment, the identified changes in the quality of life of patients remained, as evidenced by the level of values of the questionnaire.

These patients still had significantly higher baseline parameters the scales "PL - limitation of physical activity", "AF - frequency of angina pectoris" and the final indicator. The final indicator of the questionnaire was 52\% higher than the initial level (Figure 2).

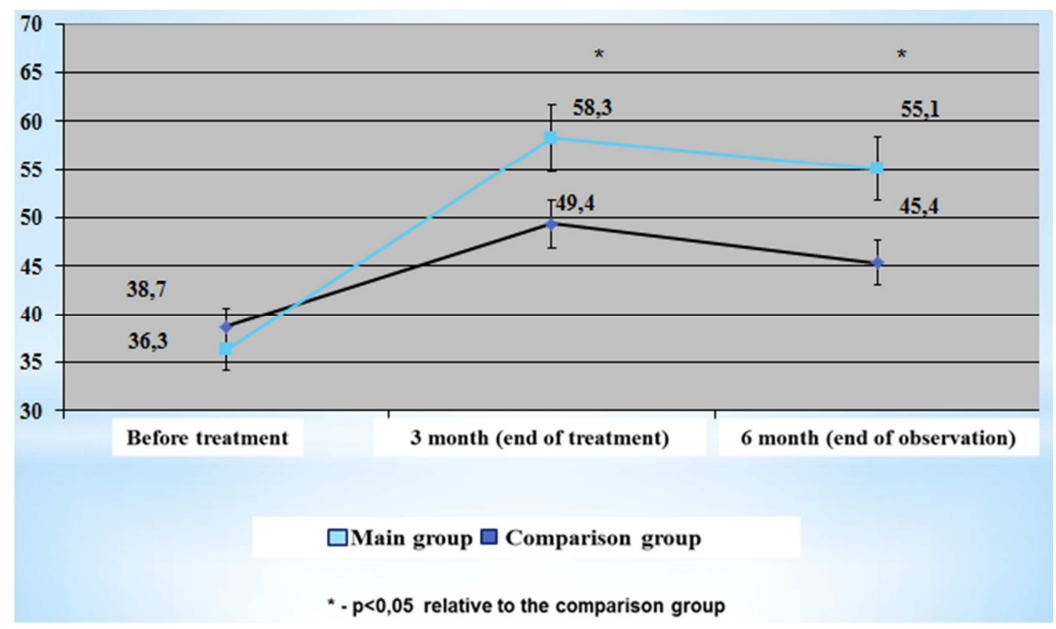

Figure 2. The dynamics final indicator of the Seattle questionnaire quality of life in the main group and the comparison group.

At the same time, the assessment of the dynamics the quality of life in the main group, who took taurine, showed that the results of most scales were significantly $(p<0.05)$ higher than the initial level, and compared with corresponding indicators in the comparison group. The values of the indicators of the questionnaire scales in patients of the main group were significantly $(p<0.05)$ above the baseline values.

Thus, the treatment provided improved the quality of life of patients in both groups, but at the same time, more pronounced dynamics was observed in patients who received taurine. Identified differences were significant after 3 months after initiation of therapy. During this period of observation, such indicators quality life as physical activity, stability of angina pectoris, frequency of strokes and satisfaction with treatment were significantly $(p<0.05)$ higher in the group patients who received taurine, than in patients of the comparison group. After 6 months the differences between the indicators quality life in the studied groups were less pronounced. However, even during this period, statistically significant differences remained on all scales.

Evaluation of the results of the treadmill test using the modified Bruce protocol revealed an increase in the level of exercise tolerance in patients of both groups, while the differences between the groups in terms of the test score after treatment were not statistically significant $(p>0.05)$. In the main group, the value of this 3 months later since the start of treatment, $7.03 \pm 0.45$ to $7.54 \pm 0.36 \mathrm{MET}$, and in the comparison group from $7.47 \pm 0.33$ to $7.99 \pm 0.25$ MET, however, the values in both groups are statistically not significantly different from baseline levels (Figure 3).

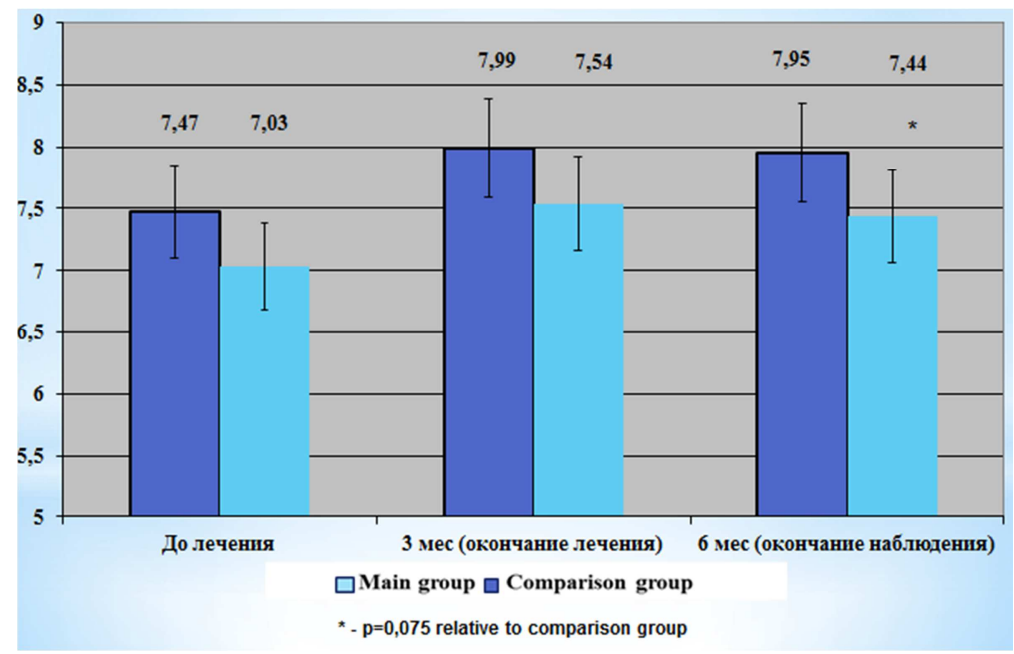




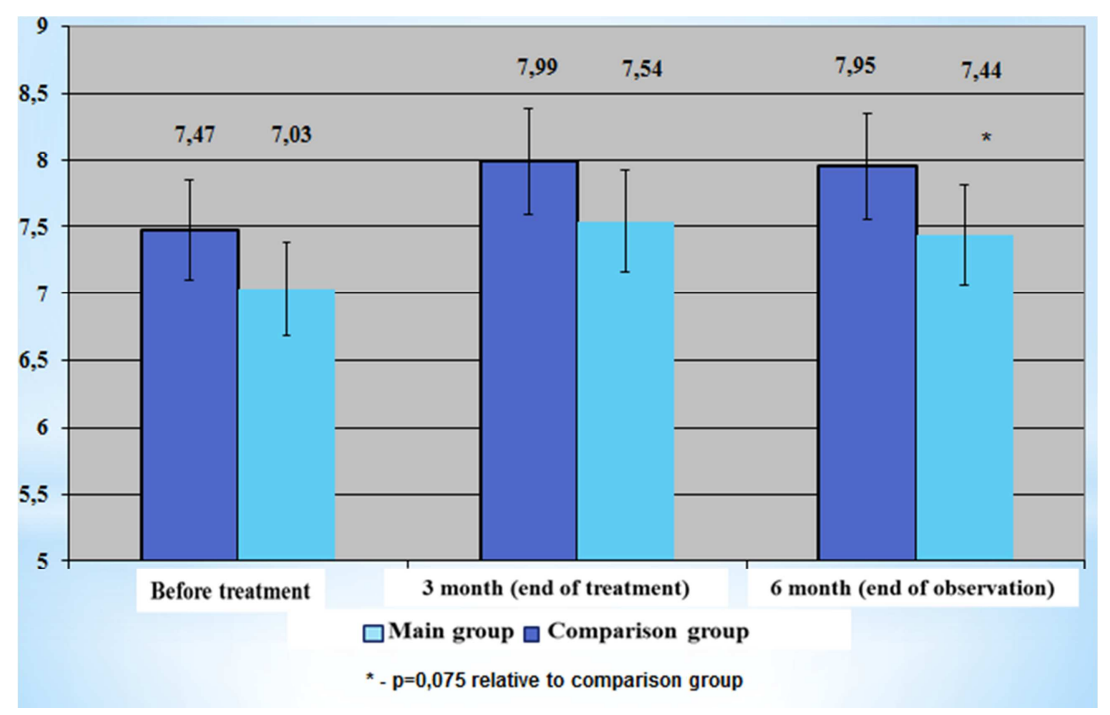

Figure 3. Dynamics tolerance to exercise in patients the main group and the comparison group, according to the results of treadmill test.

After 6 months observations in the comparison group, the results of the treadmill test remained almost the same $(7.95 \pm$ $0.21 \mathrm{MET})$, which was also noted in patients of the main group, where the value of this indicator was $7.44 \pm 0.34$ MET. At the same time, significant differences after 3 months after the end of treatment, it was not revealed both between the indicators of different groups and relative to the level of this parameter before the start of treatment $(\mathrm{p}>0.05)$.

The study dynamics of cardiac rhythm disorders in the examined patients showed that after the treatment, the average number of ventricular extrasystoles per day in the main group significantly decreased by $31.7 \%$, from $483.7 \pm$ 90.4 to $330.8 \pm 49.1$ ( $\mathrm{p}<0.005$ ) (Figure 4$)$. After 6 months the value of this indicator in patients of this group continued to decline - up to $280.4 \pm 45.7$ per day, which was also significantly lower than before the start of treatment $(\mathrm{p}$ $<0.01$ ). In this case, in the comparison group the number of ventricular extrasystoles within 3 months observations remained almost unchanged and amounted to $432.9 \pm 87.1$ at the beginning of the study $426.5 \pm 79.0$ after 3 months. After 6 months from the beginning of the study the number of ventricular extrasystoles in patients comparison group decreased to $331.8 \pm 63.0$, while the identified fluctuations did not have statistical significance $(p>0.05)$. At the same time, the value of this indicator after treatment was significantly higher $(\mathrm{p}<0.05)$ than in patients whose treatment included taurine.

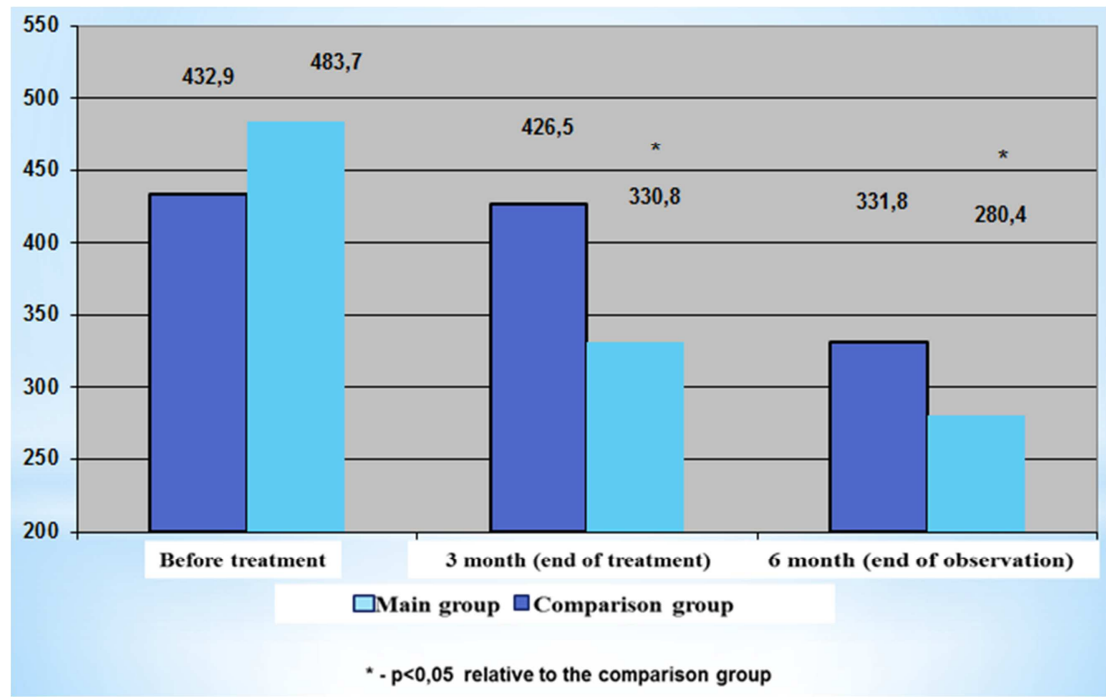

Figure 4. Dynamics number of ventricular extrasystoles per day according to Holter monitoring.

Evaluation the number of supraventricular extrasystoles per day showed that after 3 months after the start of treatment in the main group, the number of supraventricular extrasystoles significantly decreased $(\mathrm{p}<0.001)$ by $64.7 \%$, from $552.8 \pm 74.6$ to $195.2 \pm 35.7$ per day. Subsequently, the value of this indicator in patients in this group increased slightly (to $280.4 \pm 37.3$ ), but was still lower than before the start of treatment $(p<0.01)$.

In patients who took placebo, the number of supraventricular extrasystoles after treatment was slightly decreased after 6 months after the start of the study, the 
average number of supraventricular extrasystoles in the main group was significantly lower than in patients of the comparison group both after 3 months and after 6 months after the start of treatment ( $p<0.01$ in both terms).

Comparison of the echocardiographic parameters after the end of treatment in the groups of examined patients showed that patients of the main group were slightly higher than in the comparison group levels of end systolic size of the left ventricle, end diastolic size of the left ventricle and end diastolic volume of the left ventricle. At the same time, the values of end systolic volume and ejection fraction were, on the contrary, higher in the patients of the comparison group, although there were no significant intergroup differences in all compared parameters (Table 2). In patients taking taurine, the value of the ejection fraction was significantly higher $(\mathrm{p}<0.05)$ than in the comparison group, respectively, $56.0 \pm 1.8$ and $53.5 \pm 1.1 \%$.

Table 2. Dynamics left ventricular echocardiography indicators in the examined patients, $M \pm m$.

\begin{tabular}{llll}
\hline \multirow{2}{*}{ Indicators } & Before treatment & After 3month treatment \\
\cline { 2 - 4 } & Comparison group $(\mathbf{n = 4 7 )}$ & Main group $(\mathbf{n = 4 8 )}$ & Comparison group (n=47) \\
\hline Endsystolicsize, $\mathrm{mm}$ & $34,8 \pm 1,2$ & $34,4 \pm 2,1$ & $34,3 \pm 1,6$ \\
Enddiastolicsize, $\mathrm{mm}$ & $51,0 \pm 1,8$ & $51,3 \pm 2,0$ & $52,7 \pm 2,2$ \\
Endsystolicvolume, $\mathrm{ml}$ & $47,8 \pm 1,5$ & $47,2 \pm 1,7$ & $47,1 \pm 1,1$ \\
End diastolic volume, $\mathrm{ml}$ & $101,1 \pm 2,7$ & $101,5 \pm 2,0$ & $103,0 \pm 2,2$ \\
Ejection fraction, \% & $52,9 \pm 1,2$ & $52,7 \pm 1,4$ & $53,5 \pm 1,1$ \\
Stroke volume, \% & $53,8 \pm 1,1$ & $54,2 \pm 1,6$ & $54,2 \pm 1,6$ \\
\hline
\end{tabular}

Note: $*$ - the differences are significant $($ at $\mathrm{p}<0.05)$ relative to the indicator of comparison groups $(\mathrm{Cr}$. Mann-Whitney).

\section{Discussion}

Our study confirmed the findings of numerous studies on the biological activity of taurine, its ability to exert a beneficial effect on the condition of patients with cardiovascular diseases. To date, it has been established that taurine eliminates vasoconstriction caused by various causes, protects the endothelium, modulates platelet aggregation, and exhibits hypotensive effects at elevated blood pressure. The data obtained in our study are consistent with the results of other authors and confirm that in order to increase the effectiveness of treatment of the patient population in question, it is advisable to use drugs containing taurine for the treatment of patients with postinfarction cardiosclerosis.

\section{Conclusion}

The results of studies presented in the literature confirm the possibility of using taurine in the prevention of a number of cardiovascular diseases [7, 10, 11]. It has been established that the wide spectrum of action of taurine is due to its metabolic nature and the regulating effect of this compound on the functional state of the organs and body systems, as well as various types of metabolism [6-8, 10].

Our studies have confirmed its clinical efficacy when used in patients with stable exertional angina and postinfarction cardiosclerosis. The results indicate significant changes compared with placebo indicators of subjective status and quality life of these patients. In the subjective status of the surveyed, a decrease in the severity of fatigue by 2 times was noted, a decrease in the intensity of palpitations complaints by $72.3 \%$, a decrease in the severity of shortness of breath by $30.0 \%$ and a severity of pain in the heart area by $50 \%$. Indicators of the majority of scales of the Seattle questionnaire in patients of the main group at the end of the study significantly exceeded $(p<0.05)$ the corresponding values in patients of the comparison group (PL - limitation of physical activity by $32 \%$, AS - stability of stenocardia by
$20 \%$, AF $-17 \%$ and TS $-37 \%$ satisfaction with treatment).

In patients in whose treatment course taurine was used, there was a significant increase in the ejection fraction (in the main group $56.0 \pm 1.8 \%$, in the comparison group $53.5 \pm$ $1.1 \%, \mathrm{p}<0.05)$ and a tendency to a decrease in the value of the final systolic volume of the left ventricle (main group $45.3 \pm$ $1.3 \mathrm{ml}$, in the comparison group $47.1 \pm 1.1 \mathrm{ml}, \mathrm{p}>0.05$ ).

The results of the study confirmed that the use of taurine in the complex treatment of patients with stable angina and postinfarction cardiosclerosis is safe and not accompanied by side effects.

The data obtained indicating a positive effect of taurine on the clinical and instrumental characteristics of patients with postinfarction cardiosclerosis. Taurine is advisable to include in the complex treatment of patients with angina pectoris after myocardial infarction in order to improve the myocardial inotropic function, enhance antiarrhythmic and lipid-lowering effects of basic therapy. The recommended dose of taurine is $750 \mathrm{mg}$ per day as a supplement to the standard therapy. Duration of therapy at least 3 months.

\section{References}

[1] Van de Werf F, Crea F. The year in cardiology 2014: acute coronary syndromes. Eur. Heart J. 2015; 36: 342-6. DOI: 10.1093 / eurheartj / ehu488. Epub 2015 Jan 2.

[2] Nayyar S, Wilson L, Ganesan A, et al. Electrophysiologic features of protected channels in late postinfarction patients with and without spontaneous ventricular tachycardia. J. Interv. Card Electrophysiol. 2017; Dec 13. DOI: 10.1007 / s10840-017-0299-6. [Epub ahead of print].

[3] Cacciapuoti F, Tirelli P. Left ventricular postinfarctionseudoaneurysm: Diagnostic Advantages of Three-Dimensional Echocardiography. J CardiovascEchogr. 2017; 27 (2): 74-6. DOI: 10.4103 / jcecho.jcecho_49_16.

[4] Allo SN, Bagby L, Schaffer SW. Taurine depletion, a novel mechanism for cardioprotection from regional ischemia. Am. J. Physiol. 1997; 273: 1956-61. 
[5] Dyakova NA, Puzyreva IN, Ogaj MA, et al. Development of dosage forms with taurine. Bulletin of Voronezh State University. Series: Chemistry. Biology. Pharmacy. 2016; 1: P. 140-6.

[6] Doskina EV. What is taurine and "with what it is eaten"? Questions of dietetics. 2015; 5 (1): 58-61.

[7] Nechaeva GI, Druk IV, Ryapolova EA. Efficacy and tolerability of taurine in patients with type 2 diabetes mellitus and left ventricular diastolic dysfunction. Polyclinic. 2015; 1: 58-62.

[8] Schaffer SW, Shimada-Takaura K, Jong CJ, et al. Impaired energy metabolism of the taurinedeficient heart. Amino Acids. 2016; 48 (2): 549-58. DOI: 10.1007 / s00726-015-2110-2.
[9] Spertus JA, Winder JA, Dewhurst TA, et al. Development and evaluation of the Seattle Angina Questionnaire: a new functional status measure for coronary artery disease. J Am CollCardiol. 1995; 25 (2): 333-41.

[10] Stacenko ME, Turkina SV, Shilina NN, Vinnikova AA. Endothelioprotective properties of taurine in patients with chronic heart failure and type 2 diabetes mellitus. Cardiovascular Therapy and Prevention. 2016; 15 (2): 38-44.

[11] Averin E. Use of taurine during rehabilitation after cardiac surgery. Adv. Exp. Med. Biol. 2015; 803: 637-649. DOI: 10.1007 / 978-3-319-15126-7_51. 